\title{
Un ejemplo de actividad de escape room sobre física y química en educación secundaria
}

\author{
Laura Tajuelo \\ Instituto de Ciencias de la Educación, Universidad Politécnica de Madrid, España. \\ lauratajumolip@gmail.com \\ Gabriel Pinto \\ E.T.S. de Ingenieros Industriales, Universidad Politécnica de Madrid, España. \\ gabriel.pinto@upm.es
}

[Recibido: 16 junio 2020. Revisado: 27 diciembre 2020. Aceptado: 28 enero 2021]

Resumen: Tras introducir la gamificación como recurso didáctico, se comentan las características de una herramienta particular de ese tipo, el escape room, y se recoge información de un caso, preparado como actividad de repaso para la asignatura de Física y Química de $3^{\circ}$ de ESO. Se le dotó de un carácter multidisciplinar, con una temática de concienciación medioambiental y con pruebas que incluían conceptos de otras asignaturas con el fin de desarrollar competencias clave. Los estudiantes, que participaron en equipo, mostraron gran implicación y se divirtieron al mismo tiempo, manifestando que les gustaría que este tipo de actividades se realizaran más a menudo.

Palabras clave: Escape Room; Física y Química; Gamificación.

An example of escape room activity about Physics and Chemistry in the secondary education

\begin{abstract}
After introducing the gamification as a didactic resource, the characteristics of a particular tool, the escape room, are discussed and information about a specific case is explained. The case was prepared as a review activity for the subject Physics and Chemistry of the 3rd course of the Compulsory Secondary Education. It was endowed with a multidisciplinary character, including environmental awareness as guiding theme, exercises that included concepts from different subjects and taking into consideration skills, attitudes and knowledge contained in key competences. Students who participated by teamwork showed great involvement and had fun at the same time. They mentioned that they would like these type of activities to be more often implemented.
\end{abstract}

Keywords: Escape room; Gamification; Physics and Chemistry.

Para citar este artículo: Tajuelo L., Pinto G. (2021) Un ejemplo de actividad de escape room sobre física y química en educación secundaria. Revista Eureka sobre Enseñanza y Divulgación de las Ciencias 18(2), 2205. doi: 10.25267/Rev_Eureka_ensen_divulg_cienc.2021.v18.i2.2205

\section{Introducción a la gamificación}

Vivimos una época en la que los estudiantes crecen desde pequeños en contacto con tecnologías y recursos informáticos que no existían en el pasado. Por ello, también presentan nuevas actitudes frente al proceso de aprendizaje que pueden requerir nuevos paradigmas pedagógicos y tendencias educativas que cubran estas características y necesidades (Furdu et al. 2017). Por otro lado, el interés de los alumnos por las ciencias puede verse mermado si no se logra despertar su curiosidad. De hecho, muchos estudiantes de Secundaria confiesan que la asignatura de Física y Química les resulta aburrida y/o difícil en comparación con otras (Méndez-Coca 2015). La estructura de una clase de esta asignatura suele consistir en introducir los términos y conceptos mediante la presentación de definiciones, seguido de una explicación o lectura de un libro de texto y, por último, se resuelven problemas tipo. Aunque parece un esquema asentado, en ocasiones los alumnos no responden bien al mismo (Yuriev et al. 2016). 
El proceso educativo debe estar sometido de manera continua a una adaptación que tenga en cuenta el uso de nuevas tecnologías y tendencias de aprendizaje para asegurar su calidad. Por ello, es importante realizar nuevas actividades que resulten ser del interés del alumnado. Una de las técnicas que merece la pena destacar al respecto es la gamificación (utilización de mecánicas de juegos en entornos no lúdicos), en la que los estudiantes pueden tener una participación más activa y disfrutar de tareas más complejas. Las actividades de gamificación han sido aplicadas a distintas áreas del conocimiento y han permitido simular situaciones a las que los estudiantes se pueden enfrentar en algún momento de sus vidas (Ferreiro-González et al. 2019). Tienen como valor añadido que permiten trabajar tanto conocimientos como el desarrollo de habilidades sociales y creativas, en un ambiente en el que tienen gran peso la motivación, la diversión y la interacción (Clarke et al. 2017). Hay características de los juegos que ayudan a crear la atmósfera deseada. Algunos ejemplos son los sistemas de recompensa, el progreso según los niveles alcanzados, la ganancia de puntos o insignias según las tareas completadas con éxito y el hacer visibles los avances de distintos participantes para estimular la competición.

En cuanto a los pros y contras de la gamificación, como señalan Furdu et al. (2017), podemos encontrar ventajas como la facilidad para captar la atención del estudiante, el hecho de plantear retos que puedan conseguir que se alcancen objetivos de aprendizaje, la creación de un espacio adecuado con un ambiente positivo y dinámico, el valor añadido de la diversión y el fomento de una actitud participativa. Entre los inconvenientes se destacan los casos en los que algún alumno tenga miedo a fallar y derive en desmotivación y frustración. Es importante fomentar una actitud adecuada para que sepan ver los fallos cometidos como "oportunidades para aprender". Por eso, un paso previo fundamental será diseñar las tareas de manera que, cuando haya un error, exista la oportunidad de poder volverlo a intentar.

Un juego aplicado a la educación que cuente con un buen diseño debe conseguir que la motivación alcanzada durante el mismo perdure al salir del aula. Esta motivación dependerá del perfil de los alumnos. Pese a resultar atractivo para los más competitivos, también habrá estudiantes que no lo sean y esto les genere una falta de interés, así como participantes que pierdan la motivación cuando su posición esté alejada de los primeros puestos. Así, la gamificación puede ser una herramienta complementaria poderosa y útil para guiar un proceso de aprendizaje, siempre que se tenga en cuenta el contexto, los objetivos que se persiguen y otros factores que influyen en el resultado de la actividad (Stott y Neustaedter 2013).

Los objetivos de este trabajo son: analizar las características generales del escape room como un caso de herramienta educativa complementaria de gamificación; presentar una experiencia concreta que se desarrolló para el aprendizaje de conceptos de física y química y otras materias a nivel de Educación Secundaria; aportar ideas sobre su preparación e implementación y analizar los resultados, basados principalmente en un cuestionario final cubierto por los participantes. Todo ello con la idea de que pueda ser de inspiración para el profesorado de distintas etapas educativas.

\section{Marco teórico y antecedentes: características del escape room}

El escape room (a veces traducido como "sala de escape") ha sido definido como un juego en vivo basado en equipos (live-action team-based game) o un juego de lógica (Ferreiro-González et al. 2019) donde los participantes tienen que descubrir pistas, buscar objetos perdidos, resolver puzles y adivinanzas, desvelar misterios, prestar atención a los detalles o llevar a cabo tareas en una o más habitaciones con el fin de conseguir un objetivo específico (normalmente escapar de la habitación en un límite de tiempo) (Hermanns et al. 2017). Suelen realizarse en grupos pequeños de no más de seis personas. También existe en los entornos virtuales como video- 
juego o aplicación. En los últimos años, han tenido mucho éxito como experiencias mentales y físicas en las que la inteligencia y la rapidez juegan un papel importante, y como actividad de entretenimiento para grupos de amigos en un amplio rango de edad (Coffman-Wolph et al. 2018).

Un escape room puede ser temático y guiar su desarrollo a través de una historia capaz de crear una atmósfera que fomente la curiosidad y proporcione un rol a los participantes, de manera que adquieran una responsabilidad en la misión de completar las tareas a tiempo. La temática puede resultar un elemento de gran interés para algunos participantes e irrelevante para otros que simplemente se centren en la resolución de los retos. Las historias que más se repiten están relacionadas con atracos, prisiones, accidentes de laboratorio, resolución de un crimen o misterio, desconectar explosivos, espionaje, encontrar a personas desaparecidas, ayudar a crear una cura, sobrevivir a una situación o llevar a cabo una operación militar (Coffman-Wolph et al. 2018, Nicholson 2015). No hay un modelo exacto que ofrezca una respuesta de éxito total. Por eso, será importante coger experiencia en la organización de la actividad para valorar, lo mejor posible, la manera de adaptarla a cada contexto.

A nivel educativo, algunos factores que hay que tener en cuenta son: el tipo de participante, el tiempo (desde quince minutos a varios días), la dificultad, el modo de juego (cooperativo, competitivo o mixto) y el número de jugadores. Para conseguir que la actividad sea efectiva, el docente también debe prestar atención a tener un feedback rápido, a considerar el "derecho a fallar" de los alumnos, a la progresión y a la historia que envuelve la temática escogida. Si el equipo se estanca, siempre debe existir alguna manera que les permita salir de ese estado para continuar resolviendo los misterios. Los participantes practican lo aprendido y si el feedback es dado de manera frecuente y acertada, podrán volverse más autodidactas en el proceso (Stott y Neustaedter 2013).

La manera de organizarlo puede basarse en distintos criterios. Se puede seguir un orden determinado mediante unos pasos y creando una secuencia que conduce al resultado final o tener una estructura más abierta en la que los jugadores pueden ir resolviendo tareas que son partes de la solución final a la que deben llegar. También existen organizaciones híbridas que combinan ambas formas. Para que los participantes sean conscientes del tiempo que les queda, puede existir un reloj dentro del espacio de juego. Las pruebas pueden ser muy variadas y tener un sentido independiente o estar interconectadas. Las más populares tienen que ver con cálculos matemáticos, retos visuales o espaciales, interpretación de mapas, lógica, búsqueda de objetos o detalles en la sala, codificaciones, utilización de símbolos, resolución de problemas de tipo out of the box thinking (debe pensarse de manera distinta a la convencional para resolverlos) para fomentar las soluciones creativas, puzles, patrones, acertijos, sudokus, pensamiento estratégico con pruebas de ajedrez, agilidad física, interacción con actores que tengan un papel dentro de la habitación y otros retos que incluyen el uso de espejos, imágenes, sonidos, olores o el tacto. A veces, también existen pruebas que requieren del uso de un ordenador.

El inicio del escape room es un momento importante y también es fundamental poder contar con un elemento que involucre a los participantes y capte su atención. Como en cualquier otra actividad educativa, habrá que tener en cuenta la llamada curva de interés y, en ella, el comienzo es uno de los puntos en los que conviene enfatizar. Así, un escape room puede comenzar presentando a la persona que va a actuar como Gamemaster o director del juego, que introducirá las normas. Si hay una historia involucrada, puede contarse en forma de vídeo, texto o mediante actores y actrices que representen un papel dentro de la misma, y se encuentren representando un rol dentro de la habitación para que los participantes interaccionen, con el fin de obtener información para completar las misiones. También 
pueden ser meros elementos de tensión o los narradores de la historia al inicio y permanecer después como personas dispuestas a ofrecer ayuda si la requieren los equipos.

La mayoría de los escape room introducen un relato para facilitar que los participantes aprendan mejor por el hecho de estar involucrados. De hecho, cuando pasan a ser parte de la aventura y adquieren un rol de personaje responsable en la trama, participan de una manera más activa y quedan inmersos en la actividad para disfrutar de la misma. El director de juego suele ser el responsable de asegurar que todas las pruebas y materiales funcionen correctamente y estén disponibles para posibilitar el desarrollo de la actividad conforme a la previa planificación. También puede ayudar en ciertos momentos a los jugadores si se encuentran estancados. En algunos casos no está presente en la sala sino que está monitorizando las acciones a través de una cámara y, por lo tanto, la comunicación podrá ser establecida mediante ordenador u otro dispositivo. Otra función será asegurar que los participantes no dañen las instalaciones, así como actuar en caso de emergencia.

\section{El escape room como herramienta educativa}

Para que en un escape room la experiencia tenga un sentido educativo, los objetivos de aprendizaje tendrán un gran peso a la hora de diseñar la actividad. Dichos objetivos pueden estar centrados en conocimientos o desarrollo de habilidades como el liderazgo, la comunicación, el trabajo en equipo, la toma de decisiones y la resolución de problemas. Su aplicación es capaz de crear un espacio seguro para que los alumnos den rienda a los distintos estilos de resolución de problemas que forman parte de su proceso de aprendizaje. También conviene enfatizar en el potencial artístico y creativo que tiene para todas las personas involucradas. Se trata de un método que puede ser adaptado a distintos contextos educativos e incorporar contenidos de asignaturas y que, además, resulta cercano a nuestra tendencia natural de aprender mediante el juego.

El trabajo en equipo es una de las claves para resolver los misterios y finalizar la misión. De esta manera, los estudiantes establecen una colaboración entre ellos, hay sentimiento de interdependencia positiva e interacciones sociales que desarrollan actitudes de solidaridad, igualdad, respeto, diálogo y libertad (Méndez-Coca 2015).

En un ejemplo concreto reciente, titulado Escape ClassRoom "CSI 1.0" los participantes tuvieron que investigar un crimen jugando el rol de químico forense (Ferreiro-González et al. 2019), y se utilizó como ejercicio de evaluación de conocimientos químicos. Los participantes fueron 43 estudiantes universitarios. Se observó cómo los grupos compuestos por un menor número de integrantes (seis) funcionaron mejor que los integrados por nueve o diez. Otra experiencia que cabe destacar es la desarrollada en EEUU con fines educativos para alumnos de entre diez y once años con el objetivo de repasar contenidos del curso y llevar a cabo una actividad que consiguiera desarrollar el razonamiento espacial y la capacidad de resolver problemas (Coff-man-Wolph et al. 2018).

A medida que los participantes van explorando la sala, encontrando pistas y resolviendo tareas, se ha observado cómo la actitud y el estado de nerviosismo se van agitando a medida que transcurre el tiempo. Con esta situación, la comunicación entre los componentes del equipo pasa a ser crucial para superar los retos con mayor rapidez y facilidad.

Aunque depende del diseño, un escape room suele ser de una sola participación ya que, una vez se haya resuelto, no tiene sentido volver a realizarlo conociendo las respuestas. Por eso, algunas personas que trabajan con este método tienen como gran reto poder crear algo que sea más fácil de repetir y reproducir, para ello las claves serían contar con distintos puzles 
generados mediante un ordenador o crear un escape room que tenga diferentes posibles soluciones.

En ocasiones, se introduce la creación de clasificaciones con los equipos que han sido más rápidos, pero hay que tener cuidado con no poner un énfasis excesivo en este detalle debido a que las reacciones de los grupos pueden ser de desmotivación. Así, una opción que puede ser muy interesante es que los participantes puedan elegir qué tipo de juego quieren, si competitivo o no competitivo, para ayudar a establecer características que se adapten a sus deseos y necesidades con el fin de contribuir a una experiencia más personalizada (Nicholson 2015). Otro modo de prevenir estos puntos es planificar con suficiente tiempo la actividad, comprobar cuánto se tardará en resolver cada tarea, revisar y redefinir instrucciones, crear un escenario con el ambiente adecuado, asegurar los objetivos, avisar a los alumnos de que pueden encontrarse retos de mayor dificultad entre las pruebas y crear una atmósfera de actitud positiva (Hermanns et al. 2017).

Crear un pequeño momento al final del escape room para volver emocional y mentalmente al "mundo real" es importante teniendo en cuenta que la actividad envuelve dos estados diferentes, así que un enfriamiento al final de una situación que ha podido generar estrés resulta interesante, también para ofrecer un espacio en el que, por un lado, se hable de cómo ha ido la experiencia y, por otro, se pueda recibir un feedback que ayude al organizador.

\section{Preparación e implementación de un caso de escape room educativo}

Ante la baja frecuencia con la que se implementan este tipo de métodos en las aulas y con la motivación de ofrecer a los alumnos una sesión distinta que se llevara a cabo de manera dinámica, con trabajo en equipo, participación activa y que pudiera dar cabida a otras formas de aprender, surgió la idea de aprovechar la tendencia del escape room y aplicarlo en este contexto de educación formal. Así, el denominado Escape ClassRoom Save the Water fue una idea propuesta por la coautora de este trabajo, como actividad de repaso de contenidos para la asignatura de Física y Química de $3^{\circ}$ ESO. Se realizó en un colegio de Madrid capital en abril de 2019, con 6 clases de cerca de 20 alumnos cada una y el apoyo de otros profesores del centro. No formó parte de la evaluación de la asignatura. Los objetivos de la actividad se basaron en los contenidos, criterios de evaluación y estándares de aprendizaje del bloque 3 "Los cambios" y del inicio del bloque 4 "El movimiento y la fuerza" (Orden ECD/65/2015). Sin embargo, la intención era ir más allá para dotar la sesión de un carácter multidisciplinar y para ello, se incluyeron pinceladas de contenidos recientes del resto de asignaturas, así como se tuvieron muy presentes las competencias clave y la educación en el desarrollo de capacidades, valores y actitudes que persiguen las mismas. Mediante la aplicación de este método también se pretendió observar la reacción de los estudiantes, evaluar si el carácter multidisciplinar era de su interés y conocer si la actividad era bien recibida, entre otros aspectos.

El primer paso para preparar la actividad fue tener claros los otros contenidos que se iban a incluir y para ello fue necesario hablar con los profesores de otras asignaturas para conocer los conceptos que estaban trabajando. En Tecnología estaban aprendiendo a hacer diseños para impresión 3D, en Música la identificación de instrumentos de cuerda, en Lengua Castellana y Literatura analizaban El Quijote, en Geografía e Historia iniciaban el sector terciario y el agua como recurso, en Biología y Geología, las enfermedades, y en Educación Física practicaban natación y baloncesto. Las competencias clave estarían presentes a través de: la comunicación en un contexto de interacción social en el que se plantea el trabajo en equipo; el fomento de la actitud investigadora; la resolución de problemas; la capacidad de razonamiento y la de realizar interpretaciones adecuadas; la concienciación medioambiental; el fomento de un estilo de vida saludable; el uso de recursos digitales e informáticos; aprender a aprender mediante el 
contacto con materiales y metodologías poco frecuentes bajo el marco de la educación formal; el sentido de la iniciativa y el liderazgo; la creatividad; la línea de trabajo colaborativa; y la presencia de aspectos culturales e históricos. Por supuesto, todo esto conlleva también el fomento y el desarrollo de una serie de actitudes positivas y valores entre los que se podrían mencionar el respeto y la tolerancia. Una vez que se recogió toda la información necesaria, el siguiente paso consistió en organizarla y combinarla para plantear las pruebas que, en su conjunto, darían lugar a la actividad.

El agua apareció como punto común entre algunas de las asignaturas. Como valor añadido, también puede tratarse desde el punto de vista medioambiental y como un compuesto fundamental para la vida y la salud. Por otro lado, los instrumentos de cuerda y El Quijote aparecerían incluidas como parte de las pruebas a resolver. La asignatura de Inglés se utilizaría directamente como lengua en algunas partes y la de Matemáticas se consideraría involucrada a la hora de hacer los cálculos de los problemas.

Gracias a la colaboración del profesor de Tecnología, se hizo para cada alumno un modelo de molécula de agua con impresora 3D como detalle final de la actividad y para dar sentido a la misión que los estudiantes tendrían que resolver. Dos semanas antes de la implementación, se informó a los estudiantes de que iban a participar en un escape room para repasar la materia, noticia que recibieron con sorpresa, excitación y una actitud bastante positiva.

Finalmente, la historia que conduciría el Escape ClassRoom Save the Water consistiría en una misión en la que los estudiantes tomarían el rol de científicos seleccionados específicamente para intervenir en la misma. El resumen del argumento es el siguiente: debido a que las moléculas de agua se encuentran en constante amenaza por culpa del uso irresponsable y contaminación provocados por el ser humano, se las ha retenido hasta que se demuestre que las personas comprendan su importancia y sean capaces de asumir la responsabilidad que les corresponde; los alumnos deben resolver distintas pruebas para liberar a las preciadas moléculas y poder salir de la clase.

La actividad requirió de la creación de diversos materiales y recursos que permitieron desarrollar una experiencia adaptada a las necesidades y a los objetivos formulados que se pretendían alcanzar. Para ello, se utilizaron objetos cotidianos, pero también hizo falta realizar una inversión en papelería y comercios análogos. El coste total fue de unos $50 €$. Esta fase de creación de materiales y recursos fue laboriosa al tratarse de un trabajo manual en su mayoría; también fueron necesarias otras herramientas digitales y programas de edición de imagen, audio y vídeo.

La manera de introducir la temática fue mediante un vídeo de 5 minutos, accesible en https://www.dailymotion.com/video/x7u6n0k. Allí se informa del argumento que justifica que las moléculas de agua -uno de los mayores tesoros de los que disponemos- están retenidas en una caja que los alumnos tienen que desbloquear, resolviendo una serie de retos. Los cofres de cada equipo se realizaron con cajas de cartón forradas con papel plateado, y con agujeros para crear un sistema de cierres con alambres unidos por bridas que cortarían los participantes con tijeras cada vez que indicaran la clave correcta obtenida en cada prueba. Cada vez que se cortaba una brida para desbloquear la caja se señalaba en la tabla de progreso de cada equipo. Al resolver todas las pruebas, los participantes encontraban otra caja en el interior con una llave. Los alumnos se daban cuenta de que para finalizar la actividad con éxito debían cooperar todos los compañeros de clase, porque se necesitan las cuatro llaves de los cuatro equipos para abrir el cofre final. 
En la figura 1 se recogen algunas fotografías con detalles de la actividad, donde se abordaron los contenidos resumidos en la tabla 1.

Tabla 1. Contenidos involucrados en cada prueba.

\begin{tabular}{|c|l|l|}
\hline Prueba & Contenidos de Física y Química & Contenidos de otras asignaturas \\
\hline 1 & Ley de conservación de la masa (Lavoisier). & $\begin{array}{l}\text { Historia: la revolución francesa. } \\
\text { Matemáticas: realización de cálculos. }\end{array}$ \\
\hline 2 & $\begin{array}{l}\text { Influencia de la temperatura en la velocidad de las } \\
\text { reacciones químicas. } \\
\text { Experiencia práctica y materiales de laboratorio. }\end{array}$ & $\begin{array}{l}\text { Biología y Geología: la salud y el papel del } \\
\text { agua en la transmisión de enfermedades y } \\
\text { como medio en el que preparar } \\
\text { medicamentos. }\end{array}$ \\
\hline 3 & $\begin{array}{l}\text { Número de moles, número de moléculas y } \\
\text { constante de Avogadro. }\end{array}$ & Matemáticas: realización de cálculos. \\
\hline 5 & $\begin{array}{l}\text { Cálculo de velocidad media. } \\
\text { representación de reacciones químicas. } \\
\text { Ajuste estequiométrico. } \\
\text { Características de la molécula de agua. }\end{array}$ & $\begin{array}{l}\text { Biología y Geología: deporte y salud. } \\
\text { Educación Física: la natación. }\end{array}$ \\
\hline 6 & $\begin{array}{l}\text { Reconocimiento de modelos moleculares y } \\
\text { fórmulas químicas. }\end{array}$ & $\begin{array}{l}\text { Música: identificación de instrumentos de la } \\
\text { familia de cuerda. }\end{array}$ \\
\hline 7 & $\begin{array}{l}\text { Interpretación de la tabla periódica, números } \\
\text { másicos y atómicos }\end{array}$ & $\begin{array}{l}\text { Lengua y Literatura: El Quijote. } \\
\text { Geografía e Historia: el agua como recurso. }\end{array}$ \\
\hline
\end{tabular}

De forma escueta, el conjunto de pruebas fue el siguiente:

- Prueba 1. Tras introducir brevemente la labor de Lavoisier, el reto es descubrir el año en que falleció guillotinado. Para ello, se tiene que resolver un problema de conservación de masa (combustión de metano), se recibe una linterna que ilumina a través de un agujero una caja oscura (con una tapa con forma de gota de agua gigante), y se observa la fecha señalada con rotulador fluorescente.

- Prueba 2. Se debe observar dónde se disuelve antes un comprimido efervescente, en un vaso de precipitados con agua caliente o en otro con agua fría.

- Prueba 3. Se deben calcular los moles y moléculas de agua que hay en 54,0 g de esta sustancia. El problema se plantea en la parte de atrás de un puzle de 8 piezas que forman el retrato de Amedeo Avogadro.

- Prueba 4. A través de un vídeo de Mireia Belmonte, campeona olímpica de natación, se debe calcular su velocidad media en una competición, sabiendo que la piscina es de $50 \mathrm{~m}$.

- Prueba 5. Para averiguar el ángulo que forma la molécula de agua, se plantean cálculos con los coeficientes de tres reacciones químicas que deben ajustarse.
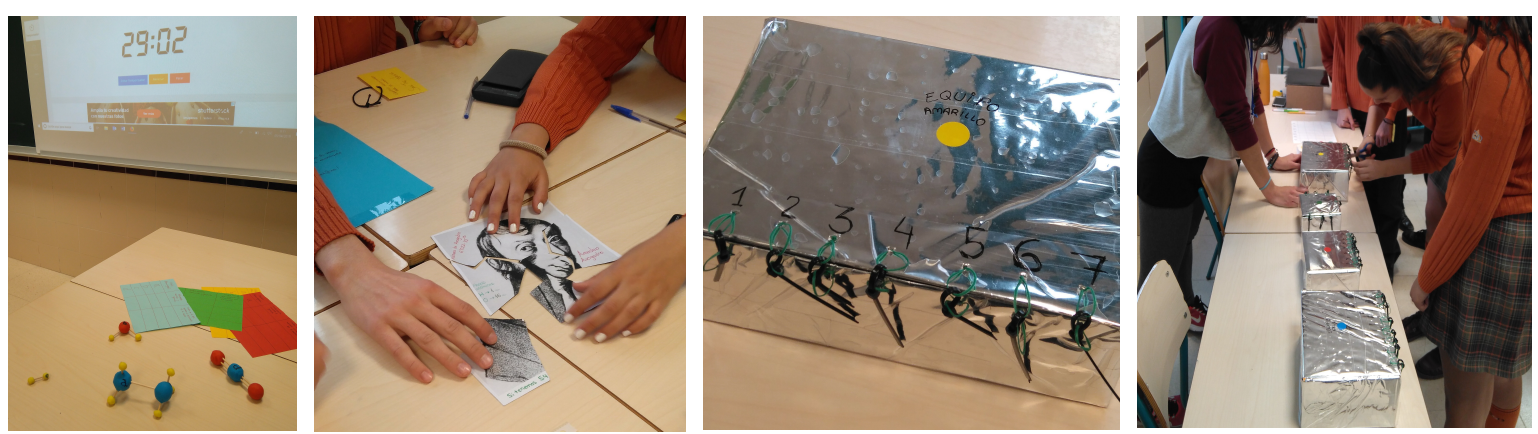

Figura 1. Fotografías de algunas pruebas y detalles del Escape ClassRoom Save the Water. 
- Prueba 6. Se oyen cuatro instrumentos (guitarra, violín, piano y arpa), que se deben colocar por orden de aparición, asociados a fórmulas químicas (realizadas con plastilina de colores y palillos) y símbolos alquimistas en una tabla (el agua lleva asociado el símbolo $\nabla$ ).

- Prueba 7. Tras introducir el molino hidráulico como fuente de energía, se debe escoger, entre varios libros, uno relacionado con molinos. Dentro de él, aparecerá una hoja con el reto de formar la palabra "molino" con símbolos de cuatro elementos cuyos números atómicos sumen 60. A partir de sus correspondientes masas atómicas se remite a una página del libro que muestra la palabra clave.

Al concluir con éxito la actividad, los alumnos encontraban este mensaje: "¡Enhorabuena grupazo! Sin vuestros conocimientos y el trabajo en equipo habría sido muy difícil llegar hasta aquí. Ahora las moléculas de agua volverán a estar libres, eso sí, no olvidéis nunca que están en nuestras manos y que pequeños actos responsables pueden dar lugar al cambio. Gracias por vuestro esfuerzo, cuidar del planeta sigue siendo nuestra misión. Cada científico puede llevarse a casa una molécula para recordarlo siempre".

Con al menos una hora de antelación, la directora del juego acudía al aula donde se iba a llevar a cabo la actividad para colocar las mesas y las sillas, distribuir los materiales y las pruebas, encender los dispositivos necesarios (proyector, tablet, ordenador y altavoces) y comprobar que funcionara todo correctamente. Los alumnos iban a su clase habitual donde dejaban todo a excepción de una calculadora y un bolígrafo. Desde ahí la profesora les acompañaba hasta el aula en la que estaba preparada la actividad.

Para empezar, la directora del juego que les recibía y les invitaba a tomar asiento, daba la bienvenida, se presentaba, introducía la actividad, contaba las normas y repartía una acreditación de científico llamado para la misión a cada alumno. Los cuatro equipos se distinguían con acreditaciones hechas de cartulina de distintos colores. A continuación, se proyectaba el vídeo que hacía de introducción para que los alumnos asumieran su rol y entendieran la historia en la que pasaban a estar involucrados. Una vez finalizado el vídeo, los estudiantes podían buscar al resto de integrantes de su equipo reuniéndose por colores según la acreditación asignada. En la pantalla del proyector comenzaba una cuenta atrás con los minutos que quedaban.

La directora del juego pasaba entonces a situarse en un extremo del aula con unas mesas sobre las que se encontraban 4 cofres para los equipos y desde donde se podía visualizar todo el espacio para monitorizar la sesión. Cada cofre estaba identificado con el color del equipo y contaba con un sistema de cierre de siete bloqueos correspondientes a las 7 pruebas. Cada vez que un equipo resolviera una prueba, debía acudir a la mesa de los cofres para decirle la clave obtenida a la "guardiana" (directora del juego). Si la respuesta era correcta, se les permitiría cortar la brida que mantuviera cerrado el bloqueo con el número de esa prueba y si, por el contrario, era incorrecta, debían volver al lugar de la prueba e intentarlo de nuevo hasta dar con la clave.

Una vez que los equipos desbloquearan por completo su cofre, cortando las 7 bridas, encontraban dentro otra caja con una llave. Entonces, buscaban el cofre final cerrado con 4 candados. De este modo, la manera de salir de la clase y de abrir esta caja final, solo era posible si todos terminaban sus pruebas. Dentro del cofre final encontraban una molécula de agua impresa en 3D para cada alumno y el mensaje en el que se les felicitaba y se les recordaba la importancia de ser responsable con respecto al uso del agua. Cada estudiante cogía su obsequio y se daba por terminada la sesión, no sin antes agradecerles la participación y 
preguntar qué les había parecido la actividad. También se les avisó de que recibirían una encuesta en los próximos días para ser parte de la evaluación de la actividad.

El Escape ClassRoom Save the Water se llevó a cabo cuando ya había terminado la unidad didáctica de reacciones químicas y habían iniciado la del movimiento. Los equipos se formaron de manera aleatoria. En total, fueron unos 120 alumnos los que pasaron por la actividad en el plazo de una semana. La actividad se planificó para ser realizada en una sesión normal de clase, es decir, en 55 minutos. Debido a que estaba dotada de cierto carácter extraordinario, podría alargarse unos minutos más con el fin de permitir que finalizara adecuadamente.

Para la directora del juego, tomaron un papel fundamental las habilidades de resolución de problemas y de adaptación ante posibles incidentes críticos o cambios inesperados. Por ejemplo, en la primera sesión el proyector no funcionó y no se pudo realizar la introducción a la temática mediante el vídeo que se había creado con dicho fin. En su lugar, la directora del juego contó oralmente de qué se trataba y dio paso al comienzo.

Las sesiones se establecieron en un aula que iba a estar disponible toda la semana, por lo que se pudo dejar montada gran parte de la actividad, de una sesión a otra, sin tener que recoger cada vez que finalizara. Para separar los residuos generados, se colocaron dos bolsas en la parte donde se encontraban los cofres con el fin de tirar los papeles en una y las bridas de plástico en otra.

\section{Valoración por los alumnos del escape room implementado}

De los cerca de 120 alumnos que realizaron la prueba, 79 contestaron un cuestionario anónimo, en la siguiente semana, cuyos resultados se resumen en la tabla 2.

Tabla 2. Resultado del cuestionario que cumplimentaron los alumnos sobre el escape room desarrollado.

\begin{tabular}{|c|c|c|c|c|c|}
\hline \multirow{3}{*}{$\begin{array}{l}\text { Cuestión } \\
\text { 1. ¿Con qué frecuencia estás en contacto con este tipo } \\
\text { de actividades en el centro educativo o en actividades } \\
\text { extraescolares? }\end{array}$} & \multicolumn{5}{|c|}{ Valoraciones } \\
\hline & Ninguna & Baja & Media & Alta & $\begin{array}{c}\text { Muy } \\
\text { alta }\end{array}$ \\
\hline & $20,3 \%$ & $60,7 \%$ & $16,5 \%$ & $2,5 \%$ & $0 \%$ \\
\hline \multirow{2}{*}{$\begin{array}{l}\text { 2. ¿Te gustaría que se llevaran a cabo con mayor } \\
\text { frecuencia? }\end{array}$} & \multicolumn{2}{|l|}{$\overline{\text { Sí }}$} & $\mathrm{No}$ & \multicolumn{2}{|c|}{ Indiferente } \\
\hline & \multicolumn{2}{|l|}{$98,7 \%$} & $0 \%$ & \multicolumn{2}{|c|}{$1,3 \%$} \\
\hline \multirow{2}{*}{$\begin{array}{l}\text { 3. ¿Crees que es una buena manera de repasar } \\
\text { contenidos de las asignaturas? }\end{array}$} & \multicolumn{2}{|l|}{ Sí } & No & \multicolumn{2}{|c|}{ Indiferente } \\
\hline & \multicolumn{2}{|c|}{$97,5 \%$} & $0 \%$ & \multicolumn{2}{|c|}{$2,5 \%$} \\
\hline \multirow{2}{*}{ 4. ¿Has aprendido algo nuevo durante la actividad? } & \multicolumn{2}{|l|}{ Sí } & $\mathrm{No}$ & \multicolumn{2}{|c|}{ Indiferente } \\
\hline & \multicolumn{2}{|c|}{$62,0 \%$} & $19,0 \%$ & \multicolumn{2}{|c|}{$19,0 \%$} \\
\hline \multirow{2}{*}{$\begin{array}{l}\text { 5. ¿Te ha resultado interesante o te ha gustado encontrar } \\
\text { contenidos de otras asignaturas además de los propios } \\
\text { de Física y Química? }\end{array}$} & \multicolumn{2}{|l|}{ Sí } & No & \multicolumn{2}{|c|}{ Indiferente } \\
\hline & \multicolumn{2}{|l|}{$73,4 \%$} & $3,8 \%$ & \multicolumn{2}{|c|}{$22,8 \%$} \\
\hline \multirow{2}{*}{$\begin{array}{l}\text { 6. ¿Te gustaría crear y preparar con tu clase un escape } \\
\text { room para otros compañeros de } 3^{\circ} \mathrm{ESO} \text { ? }\end{array}$} & \multicolumn{2}{|l|}{$\overline{\text { Sí }}$} & No & \multicolumn{2}{|c|}{ Indiferente } \\
\hline & \multicolumn{2}{|l|}{$60,8 \%$} & $15,2 \%$ & \multicolumn{2}{|c|}{$24,0 \%$} \\
\hline \multirow{2}{*}{$\begin{array}{l}\text { 7. ¿Crees que este tipo de actividades ayudan a mantener } \\
\text { o mejorar la atmósfera de trabajo y las relaciones entre } \\
\text { los compañeros? }\end{array}$} & \multicolumn{2}{|l|}{ Sí } & No & \multicolumn{2}{|c|}{ Indiferente } \\
\hline & \multicolumn{2}{|c|}{$81,0 \%$} & $3,8 \%$ & \multicolumn{2}{|c|}{$15,2 \%$} \\
\hline \multirow{2}{*}{$\begin{array}{l}\text { 8. Cuando hay que trabajar en equipo, ¿cómo prefieres } \\
\text { que se formen? }\end{array}$} & \multicolumn{3}{|c|}{ Eligiendo voluntariamente } & \multicolumn{2}{|c|}{ Aleatoriamente } \\
\hline & & $78,2 \%$ & & & \\
\hline 9. Cómo crees que aprenderías más y desarrollarías más & Eligienc & lo volunt & riamente & Aleatc & amente \\
\hline capacidades de trabajo en equipo? & & $58,1 \%$ & & & \\
\hline
\end{tabular}


Tabla 2. Continuación

\begin{tabular}{|c|c|c|c|c|c|}
\hline Cuestión & \multicolumn{5}{|c|}{ Valoraciones } \\
\hline \multirow{2}{*}{ 10. ¿Cómo trabajarías más a gusto? } & \multicolumn{2}{|c|}{ Con amigos } & $\begin{array}{l}\text { Lon equipo } \\
\text { aleatorio }\end{array}$ & \multicolumn{2}{|c|}{ Indiferente } \\
\hline & \multicolumn{2}{|c|}{$70,9 \%$} & $3,8 \%$ & \multicolumn{2}{|c|}{$25,3 \%$} \\
\hline \multirow{2}{*}{$\begin{array}{l}\text { 11. En este caso, el escape room se ha desarrollado en una } \\
\text { sesión de clase. ¿'Te gustaría que durará más tiempo? }\end{array}$} & \multicolumn{2}{|c|}{ Sí } & No & \multicolumn{2}{|c|}{ Indiferente } \\
\hline & \multicolumn{2}{|c|}{$83,3 \%$} & $6,3 \%$ & \multicolumn{2}{|c|}{$11,4 \%$} \\
\hline \multirow{2}{*}{$\begin{array}{l}\text { 12. ¿En general, te ha parecido que la información y las } \\
\text { instrucciones de las pruebas se encontraban explicadas } \\
\text { con claridad? }\end{array}$} & \multicolumn{2}{|c|}{ Sí } & Regular & \multicolumn{2}{|c|}{ No } \\
\hline & \multicolumn{2}{|c|}{$89,9 \%$} & $10,1 \%$ & \multicolumn{2}{|c|}{$0 \%$} \\
\hline \multirow{2}{*}{$\begin{array}{l}\text { 13. ¿Cómo crees que ha sido el nivel de dificultad de las } \\
\text { pruebas? }\end{array}$} & $\begin{array}{l}\text { Muy } \\
\text { fácil }\end{array}$ & Fácil & $\begin{array}{l}\text { Adecuad } \\
\text { o }\end{array}$ & Difícil & $\begin{array}{l}\text { Muy } \\
\text { difícil }\end{array}$ \\
\hline & $5,1 \%$ & $10,1 \%$ & $82,3 \%$ & $2,5 \%$ & $0 \%$ \\
\hline \multirow{2}{*}{$\begin{array}{l}\text { 14. ¿Cuál es tu valoración en cuanto a los materiales y } \\
\text { recursos utilizados en las pruebas? }\end{array}$} & $\begin{array}{l}\text { Muy } \\
\text { baja }\end{array}$ & Baja & Media & Alta & $\begin{array}{c}\text { Muy } \\
\text { alta }\end{array}$ \\
\hline & $0 \%$ & $0 \%$ & $17,7 \%$ & $58,2 \%$ & $24,1 \%$ \\
\hline \multirow{2}{*}{ 15. ¿Te ha resultado divertida la experiencia? } & \multicolumn{2}{|c|}{ Sí } & No & \multicolumn{2}{|c|}{ Indiferente } \\
\hline & \multicolumn{2}{|c|}{$92,4 \%$} & $0 \%$ & \multicolumn{2}{|c|}{$7,6 \%$} \\
\hline \multirow{2}{*}{ 16. ¿Cuál es tu valoración general de la actividad? } & $\begin{array}{l}\text { Muy } \\
\text { mala }\end{array}$ & Mala & Media & Buena & $\begin{array}{l}\text { Muy } \\
\text { buena }\end{array}$ \\
\hline & $0 \%$ & $0 \%$ & $3,8 \%$ & $43,0 \%$ & $53,2 \%$ \\
\hline
\end{tabular}

Además, se pidió opinión a los alumnos sobre algunas de las cuestiones. En la 9, al preguntarles que por qué creen que aprenden más y desarrollan más capacidades de trabajo en equipo cuando éste se elige de forma voluntaria, contestan que trabajan más cómodos, se entienden mejor, y hay más confianza. Cuando afirman preferir un equipo formado aleatoriamente, razonan que es porque con los amigos se distraen más, porque se puede aprender de otros modos de resolver problemas, y porque facilita la relación con otros compañeros.

Al preguntarles con formato abierto qué es lo que más les gustó, las respuestas más repetidas (por más del 10\% de los encuestados) fueron: las moléculas 3D del cofre final, el vídeo de introducción, poder trabajar en equipo, la organización de las pruebas, y el experimento con comprimido efervescente. Sobre si hay algo que no les gustó o que piensen que necesitaría mejorar, 53 no indicaron nada y el resto apuntó a aspectos como: debería durar más tiempo, se podrían hacer los grupos con amigos, aumentar el nivel de dificultad de alguna prueba, y aclarar algunas instrucciones.

Para finalizar, se les pidió que describiesen la sesión con una palabra y algunas de las que dejaron por escrito fueron: divertida, entretenida, diferente, lúdica, interesante, original, innovadora, apasionante, buena, emocionante, imprescindible, especial, fantástica e interactiva. También se les facilitó un espacio en blanco para añadir otros comentarios, observaciones o sugerencias adicionales al contenido de la encuesta. En esta parte manifestaron gran agradecimiento y expresaron su actitud positiva y apoyo al uso de metodologías distintas a las que se utilizan de manera habitual en las aulas. Como se ha indicado, algunos estudiantes sugirieron que se aumentase el nivel de dificultad, sin embargo, se intentó diseñar las pruebas para un nivel medio que no presentara muchas complicaciones porque la sesión tenía que 
desarrollarse en un tiempo limitado, en el que se incluían el desplazamiento de los alumnos al aula del escape room, la introducción con la explicación de normas, el vídeo y la resolución de la "misión".

Las profesoras responsables de las clases de Física y Química recibieron con buena aceptación la realización de la actividad como método de repaso y agradecieron el esfuerzo de la directora del juego. Prestaron ayuda en momentos específicos y se mantuvo una buena comunicación y relación con ellas. Les resultó curiosa la actitud y la involucración de los estudiantes, haciendo especial hincapié en algunos que habitualmente mostraban desinterés ante las clases. También pudieron observar roles y comportamientos que no se muestran tan fácilmente en otros entornos de aprendizaje, por lo que este tipo de actividades puede servir para conocer más de cerca a los estudiantes.

Conscientes de todo lo mencionado, el balance general ha sido muy positivo y resultó ser una experiencia personal y profesional muy enriquecedora.

\section{Resultados y conclusiones}

El escape room tiene una corta historia, pero actualmente es una actividad de gran éxito entre los jóvenes. Sus características generales hacen que pueda ser aplicado a un contexto de aprendizaje presentando un alto valor educativo.

Los estudiantes que participaron en esta actividad han valorado y agradecido mucho el esfuerzo y el trabajo que conlleva el diseño y la organización de la misma. En la implementación se divirtieron y mostraron una actitud activa y participativa mediante el trabajo en equipo. En la evaluación han puesto de manifiesto que les gustaría que este tipo de métodos se aplicaran más a menudo en el contexto educativo y han reflejado que piensan que también podría ayudar a mantener y mejorar tanto la atmósfera de trabajo como las relaciones entre los compañeros de clase. Mediante la adquisición de un rol como científico seleccionado para la misión y la resolución de pruebas se consiguió que el alumno adquiriera una buena autonomía e implicación en su propio proceso de aprendizaje.

Las profesoras mencionaron que alumnos que habían manifestado cierto desinterés durante el resto del curso habían cambiado totalmente su actitud cuando participaron en la sesión. También se reflexionó sobre el poder que podía tener el uso de metodologías distintas a las que se usan habitualmente para llegar a conocer más a los estudiantes en distintos contextos. La respuesta positiva de los alumnos, la actitud participativa y la motivación observada también aparecieron en mayor grado de lo que se contemplaba en las expectativas. Se esperaba observar a más estudiantes que mostraran indiferencia o que no respondieran con motivación ante la actividad. De igual manera, el análisis de los datos recogidos mediante el cuestionario ha puesto de manifiesto un impacto bastante positivo de la implementación del escape room en este contexto educativo.

Al inicio de la propuesta metodológica cabía esperar una mayor colaboración e implicación por parte del resto de los profesores de $3^{\circ} \mathrm{ESO}$, sin embargo, la gran cantidad de tareas a las que se enfrentan a diario hizo que la directora del juego se limitara a obtener la información mediante breves conversaciones y a invitarles a pasarse a ver la actividad. En contraposición, la curiosidad y el interés que la actividad despertó en algunos profesores sí que resultó mayor de lo esperado. Otro resultado que es evidente, pero que tiene un gran valor, es el de la obtención y el desarrollo de un recurso que cualquier profesor puede adaptar fácilmente a su contexto concreto.

Se consiguió disfrutar de las sesiones creando un espacio de aprendizaje diferente, seguro, divertido, cómodo y que consiguió dar respuesta a los objetivos planteados para su diseño. 
Es muy importante adaptar y ajustar todos los detalles del diseño del escape room para prevenir errores y desmotivaciones, así como para alcanzar los objetivos planteados.

Esta actividad requiere un proceso de creación de materiales para la docencia que permiten fomentar la competencia de aprender a aprender y adaptar la experiencia a las necesidades educativas correspondientes.

El balance general ha sido muy positivo y aunque requiere de un gran esfuerzo por parte de los docentes que se involucren, los buenos resultados y la valoración tan positiva que se ha recibido hacen que haya merecido mucho la pena y se recomienda como herramienta educativa complementaria. Todo ello favorece la intención de repetir la experiencia en cursos posteriores.

\section{Agradecimientos}

Se agradece la colaboración de los docentes (en especial de Esperanza Hojas y Ricardo Óscar Herrero) del Colegio Gredos San Diego de Moratalaz (Madrid). También se agradecen los apoyos recibidos de la Universidad Politécnica de Madrid a través del proyecto de innovación educativa IE1920.0502 "Fomento del aprendizaje STEAM basado en la indagación" y de la Obra Social "la Caixa" que financió el proyecto "La ciencia y la tecnología al alcance de tod@s".

\section{Referencias}

Clarke S., Peel D. J., Arnab S., Morini L., Keegan H., Wood O. (2017) EscapED: A framework for creating educational escape rooms and interactive games for higher/further education. International Journal of Serious Games 4(3), 73-86.

Coffman-Wolph S., Gray K., Pool M. (2018) Designing an escape room game to develop problem solving and spatial reasoning skills. ASEE IL-IN Section Conference. 5.

Ferreiro-González M., Amores-Arrocha A., Espada-Bellido E., Aliaño-González M. J., Vázquez-Espinosa M., González-de-Peredo A. V., Sancho-Galán P., Álvarez-Saura J. A., Barbero G. F., Cejudo-Bastante, C. (2019) Escape ClassRoom: Can you solve a crime using the analytical process? Journal of Chemical Education 6(2), 267-273.

Furdu I., Tomozei C., Kose U. (2017) Pros and cons gamification and gaming in classroom. Broad Research in Artificial Intelligence and Neuroscience 8(2), 56-62.

Hermanns M., Deal B., Campbell A. M., Hillhouse S., Opella, J. B., Faigle C., Campbell IV R. H. (2017) Using an 'Escape Room' toolbox approach to enhance pharmacology education. Journal of Nursing Education and Practice 8(4), 89-95.

Méndez-Coca D. (2015) Estudio de las motivaciones de los estudiantes de secundaria de física y química y la influencia de las metodologías de enseñanza en su interés. Educación XX1, 18(2), 215-235.

Nicholson S. (2015) Peeking behind the locked door: A survey of escape room facilities. White paper.

Orden ECD/65/2015, de 21 de enero, por la que se describen las relaciones entre las competencias, los contenidos y los criterios de evaluación de la educación primaria, la educación secundaria obligatoria y el bachillerato. BOE n 25, de 29 de enero de 2015.

Stott A., Neustaedter C. (2013) Analysis of gamification in education. Technical Report 2013042201, Connections Lab, Simon Fraser University, Surrey (Canadá).

Yuriev E., Capuano B., Short J. L. (2016) Crossword puzzles for chemistry education: learning goals beyond vocabulary. Chemistry Education Research and Practice 17(3), 532-554. 